\title{
Cayratinin, a New Anthocyanin from the Pericarp of Cayratia japonica
}

\author{
by Nariyuki IsHIKURA* and Mannen SHIBATA**
}

Received February 12, 1970

\begin{abstract}
Absuract
The ripe berries of Cayratia japonica get a dark purple color in late autumn. It was shown paper-chromatographically that their pericarp contains a single anthocyanin, which was obtained in an amorphous state. The pigment was an acylated anthocyanin which was degraded into three components, delphinidin, glucose and $p$-coumaric acid, by complete acid hydrolysis. The deacylated anthocyanin was different from each of the known delphinidin glucosides such as 3 monoglucoside, delphin (3,5-diglucoside) and eichhornin (3-diglucoside). This new anthocyanin, cayratinin, has proved to be delphinidin 3 -p-coumaroylsophoroside-5monoglucoside by the analytical procedures of oxidative degradation with hydrogen peroxide, partial hydrolysis with dilute acid, and u. v. absorption spectrophotometry.

Besides, eichhornin which was previously described as delphinidin 3-diglucoside was shown to be 3 -gentiobioside in the present experiment.
\end{abstract}

Cayratia japonica (Thumb.) Gagn. belonging to Vitaceae is an exuberant perennial vine and sometimes grows ranking over the weeds. The plant is distributed widely in Japan to the south-east of Asia, and blooms in July to August in our country. Its berry ripens in a dark purple color in late autumn. From the pericarp of this plant, the purple pigment was extracted with $1 \%$ methanolic hydrochloric acid, and the paper chromatography showed that the extract contains a single anthocyanin.

The pigment was an acylated anthocyanin which yields delphinidin, glucose and $p$-coumaric acid on complete acid hydrolysis and, besides, $p$-coumaroylsophorose on oxidative degradation with hydrogen peroxide. Partial acid hydrolysis of the deacylated anthocyanin showed that it contains 2 mols. of glucose attached to the 3- and 5-positions of delphinidin. Spectral data have also strengthened this. possibility.

Since this pigment is new to the literature, the name of cayratinin was provisionally assigned to this acylated anthocyanin, which corresponds to the structure of 3-p-coumaroylsophorosido-5-monoglucoside of delphinidin. By comparison with: awobanin (delphinidin 3-p-coumaroylglucosido-5-monoglucoside from Commelina communis ${ }^{1,2)}$ ), cayratinin has proved to be quite different from awobanin by a series of paper-chromatographic tests.

* Department of Biology, Faculty of Science, Kumamoto University, Kumamoto, Japan.

** Toyama Women's Junior College, Gankaiji, Toyama, Japan. 


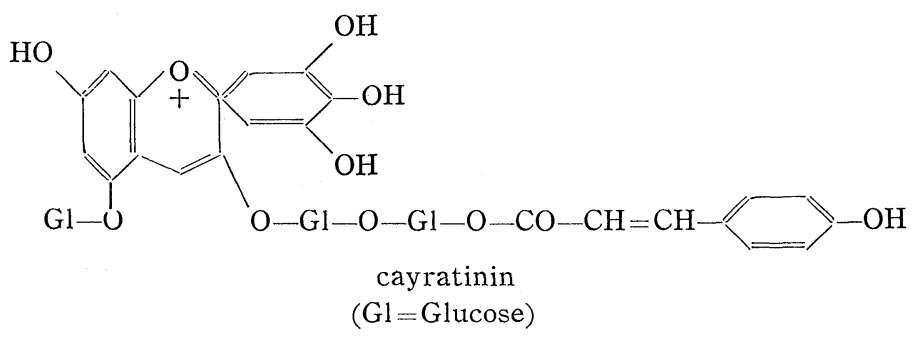

\section{Experimental Results and Discussion}

Extraction and purification of cayratinin: The ripe berries (ca. $750 \mathrm{~g}$ ) were collected from the plant, Cayratia japonica, grown on the slope of Tatsuta-yama district (Kumamoto City) in early November. The dark purple-colored, fresh pericarps (ca. $400 \mathrm{~g}$ ) were extracted repeatedly with $2 \%$ ethanolic hydrochloric acid. Paper-chromatographic tests on the crude extract showed that the color of the pericarp is due to a single anthocyanin ( $c f$., Table 1 ).

The combined purple extract (ca. 8l) was filtered, and added with methanolic solution of basic lead acetate as usual. From the lead salt of anthocyanin obtained (ca. $350 \mathrm{~g}$ ), the pigment chloride was regenerated by treatment with cold 5\% methanolic hydrochloric acid $(1 l)$, and precipitated with ether (6 vols.). An amorpoous precipitate was dissolved in $1 \%$ ethanolic hydrochloric acid $(200 \mathrm{ml})$, and the filtered solution was added with four volumes of ether. This procedure was repeated twice, and the crude anthocyanin was obtained in a state of deep purple amorphous powder. The yield was $3 \mathrm{~g}$, corresponding to $0.0075 \%$ of fresh pericarps.

All attempts to crystallization of the pigment by the use of ethanol-hydrochloric acid, and methanol-hydrochloric acid and picric acid solution were unsuccessful; rather, the pigment being more or less degraded by such treatments. The picrate separated to some extent only as a pasty mass. The pigment was soluble very easily in ethanol, methanol and water. A powdery pigment obtained above was applied on a large sheet of a thick filter paper (Tôyô, No. 526) that was previously washed with methanol and ethanol, and developed by the solvent, $n$-butanol/hydrochloric acid/water $(7: 2: 5, \mathrm{v} / \mathrm{v})(\mathrm{BuH})$. The pigment spot on the chromatogram was eluted with methanol containing hydrochloric acid in $0.1 \%$. The eluate was used for the following experiments.

Saponification of cayratinin with alkali: The above eluate was saponified by sodium hydroxide $(8 \%)$ in $80 \%$ methanol at room temperature for one hour in a stream of nitrogen. After acidification with $5 \%$ methanolic hydrochloric acid, the deacylated anthocyanin was precipitated with ether and collected. The ethereal mother liquor was evaporated to a small volume, and the organic acid liberated from the anthocyanin was obtained in the form of colorless leaflets. This acid was shown to be identical with the authentic $p$-coumaric acid by color reactions and paper chromatographic behaviors; that is, Rf-values : 0.92 in $n$-propanol/ethyl acetate/ water $(120: 20: 40, \mathrm{v} / \mathrm{v})$ (PEtAW), 0.80 in $n$-butanol/pyridine/water $(6: 3: 1, \mathrm{v} / \mathrm{v})$ (BPW), and 0.93 in $n$-butanol/acetic acid/water $(6: 1: 2, \mathrm{v} / \mathrm{v})(\mathrm{BAW})$; the color reaction being red with sulphanilic reagent ${ }^{3)}$ and fluorescent purple under $u . v$. light upon exposure to ammonia vapor.

The precipitate of deacylated anthocyanin was dissolved in $1 \%$ ethanolic hydrochloric acid, filtered from insoluble sodium chloride, and precipitated again with 
ether. After repetition of the same procedure, a reddish purple powder was obtained. The Rf value of deacylated anthocyanin in $\mathrm{BuH}$ was lower than that of the original anthocyanin (Table 1).

Another portion of the deacylated anthocyanin was further purified for the following analyses by a large-scaled paper-chromatography on Tôyô No. 526 filter paper using the solvent BuH for irrigation.

Acid hydrolysis of cayratinin: Complete hydrolysis was effected by heating for three minutes in 10\% hydrochloric acid. The solution of the hydrolysates was shaken with ether to separate the organic acid, which was identified as $p$-coumaric acid by comparison with the authentic acid in a similar manner as mentioned above.

The anthocyanidin remaining in an acidic mother liquor was extracted with iso-amyl alcohol which was saturated with 5\% hydrochloric acid. The aglycone was identified with delphinidin by means of paper chromatography (Table 1). For spectral examination, the aglycone was purified by paper chromatography using $\mathrm{AAH}$ on a large scale as above. The absorption maxima were found at 273, 335, and $547 \mathrm{~m} \mu$, which are in agreement with those of the authentic delphinidin.

The mother liquor was washed with ether to remove the last trace of $i$ so-amyl alcohol, whereupon the ether was driven away by aeration. Then the washed solution was passed through a column of Amberlite IRA 410 to remove the acid. The effluent was concentrated and examined by paper chromatography. A single spot identical with glucose was detected on any of the chromatograms, as follows: Rf 0.18 in BAW; 0.29 in PEtAW; and 0.26 in BPW. The development of the spot was effected by alkaline silver nitrate and aniline hydrochloride.

Partial acid-hydrolysates of deacylated cayratinin were also examined. For this purpose, deacylated pigment was hydrolysed with $10 \%$ hydrochloric acid by heating at $70^{\circ}$ on a water bath for two hours. Small aliquots of the reaction mixture were pipetted out at definite intervals and examined paper-chromatographically using $\mathrm{BuH}$ and also $\mathrm{AAH}$ for irrigation. In parallel, delphin (from Hyacinth ${ }^{4}$ ) was also treated, as a control, in the same way. As a consequence, three spots of partial hydrolysates were shown to be identical with the spots corresponding to 3,5-diglucoside, 3-monoglucoside and 5-monoglucoside (from delphin) of delphinidin. Rf-values of these glucosides were $0.14,0.20$ and 0.34 in $\mathrm{BuH}$, and $0.40,0.24$ and 0.24 in AAH, respectively. In this experiment, delphinidin 3-diglucoside was not clearly

Table 1. Rf-values of cayratinin, its aglycone and related compounds.

\begin{tabular}{l|ccc}
\multicolumn{1}{c|}{ Samples } & BuH* & $\begin{array}{c}\text { Rf-values in } \\
\text { AAH* }\end{array}$ & BAW* \\
Cayratinin & 0.68 & 0.62 & 0.29 \\
Deacylated cayratinin & 0.45 & 0.68 & 0.16 \\
Awobanin & 0.57 & 0.49 & 0.23 \\
Delphin & 0.14 & 0.40 & 0.01 \\
Eichhornin & 0.08 & 0.48 & 0.03 \\
Delphinidin 3-monoglucoside & 0.20 & 0.23 & 0.15 \\
Aglycone of cayratinin & 0.45 & 0.08 & - \\
Delphinidin & 0.45 & 0.08 & -
\end{tabular}

* BuH: $n$-butanol/acetic acid/water $(7: 2: 5, \mathrm{v} / \mathrm{v}), \mathrm{AAH}$ : acetic acid/hydrochloric acid/ water $(3: 1: 8, \mathrm{v} / \mathrm{v}), \mathrm{BAW}: n$-butanol/acetic acid/water $(6: 1: 2, \mathrm{v} / \mathrm{v})$. 
defined on the chromatograms. However, on the chromatogram that was run with $\mathrm{AAH}$, the spot of cayratinin was the only one that was detected at a higher position. than that of delphinidin 3,5-diglucoside. From this fact, it is strongly suggested that cayratinin is a triglucoside of delphinidin, since the Rf-value tends to increase with increasing number of sugar molecules attached to the anthocyanins, when irrigation was run with AAH.

Oxidative degradation with hydrogen peroxide: In order to clarify the mode of linkage of sugar residue and organic acid, the following experiment was carried out in the same way as described previously ${ }^{5)}$.

Three related glucosides, i.e., cayratinin, deacylated cayratinin and eichhornin (from Eichhornia crassipes $^{7)}$ ), were subjected to $\mathrm{H}_{2} \mathrm{O}_{2}$-degradation in parallel. First, the sugar moieties liberated from the latter two glucosides were examined paperchromatographically, and the spots detected on the chromatograms are summarized. in Table 2.

Next, in the case of cayratinin, an acylated sugar (refer to the product C) was. liberated by the same treatment, showing the Rf-values 0.48 in BPW and 0.42 in BAW. The color of the spot was violet-fluorescent under u.v. light $(256 \mathrm{~m} \mu)$ when exposed to ammonia vapor, and was red with sulphanilic reagent. For further examination on the product $\mathrm{C}$, the original reaction mixture was applied on a large sheet of Tôyô No 526 filter paper, and chromatographed by the use of BAW for irrigation. After elution with $70 \%$ methanol, the eluate of the product $\mathrm{C}$ was concentrated in vacuo, and subjected to saponification with $8 \%$ sodium hydroxide (in $60 \%$ methanol) in an atmosphere of nitrogen. Thus, the organic acid liberated from the product $\mathrm{C}$ was identified as $p$-coumaric acid. Another sugar moiety of the product $\mathrm{C}$ was in good agreement with sophorose in all chromatographic behaviors. Accordingly, it is evident that the product $C$ is $p$-coumaroylsophorose, whose attachment in cayratinin should have been at the 3-hydroxyl of delphinidin.

Annexed to this experiment, the exact nature of the diglucoside sugar of eichhornin was studied by means of oxidative degradation. The sugar moiety liberated by this process has proved to be gentiobiose, as shown in Table 2. Therefore, eichhornin should be defined as delphinidin 3-gentiobioside, hereafter.

Spectral examinations: All spectral measurements were made with Shimadzu

Table 2. $\mathrm{H}_{2} \mathrm{O}_{2}$-oxidation products of the deacylated cayratinin and eichhornin.

\begin{tabular}{|c|c|c|c|c|c|}
\hline Samples & $\begin{aligned} \mathrm{Rf}-\mathrm{v} \\
\mathrm{BAW}^{*}\end{aligned}$ & BPW* & $\begin{array}{l}\text { Rg-values } \\
\text { in } \\
\text { PEtAW* }\end{array}$ & $\begin{array}{c}\text { Colors } \\
\text { aniline hydrochloride }\end{array}$ & $\begin{array}{l}\text { with } \\
\text { diphenylamine- } p \text { - } \\
\left.\text { anisidine reagent }{ }^{6}\right)\end{array}$ \\
\hline $\begin{array}{l}\text { Sugar from deacy- } \\
\text { lated cayratinin }\end{array}$ & 0.09 & 0.14 & 64 & light brown & brownish orange \\
\hline $\begin{array}{l}\text { Sugar from } \\
\text { eichhornin }\end{array}$ & 0.06 & 0.06 & 45 & $\begin{array}{l}\text { grayish yellow- } \\
\text { brown }\end{array}$ & blue-green \\
\hline Sophorose & 0.09 & 0.14 & 64 & light brown & brownish orange \\
\hline Cellobiose & 0.07 & 0.09 & 55 & $\begin{array}{l}\text { grayish yellow - } \\
\text { brown }\end{array}$ & blue-green \\
\hline Gentiobiose & 0.06 & 0.06 & 45 & $\begin{array}{l}\text { grayish yellow- } \\
\text { brown }\end{array}$ & blue-green \\
\hline Glucose & 0.20 & 0.26 & 100 & brown & blue-green \\
\hline
\end{tabular}

* BAW : $n$-butanol/acetic acid/water $(6: 1: 2, \mathrm{v} / \mathrm{v}), \mathrm{BPW}: n$-butanol/pyridine/water $(6: 3: 1$, $\mathrm{v} / \mathrm{v})$, PEtAW : n-propanol/ethyl acetate/water $(120: 20: 40, \mathrm{v} / \mathrm{v})$. 
multi-purpose recording spectrophotometer MPS-50L. The pigments purified by large-scale paper-chromatography were used for this experiment throughout.

Absorption max. (in $\mathrm{m} \mu$ ) of cayratinin in $0.05 \%$ methanolic hydrochloric acid: 284, 297 (shoulder), and 535 (cf. Fig. 1). And the deacylated cayratinin possessed absorption max. (in $\mathrm{m} \mu$ ) 261, 353, and 536. The spectrum of cayratinin, unlike its deacylation product, showed a big shoulder at $297 \mathrm{~m} \mu$ which is due to the ester linkage with $p$-coumaric acid. The fact that the ester linkage lies in the sugar moiety attached to the 3 -

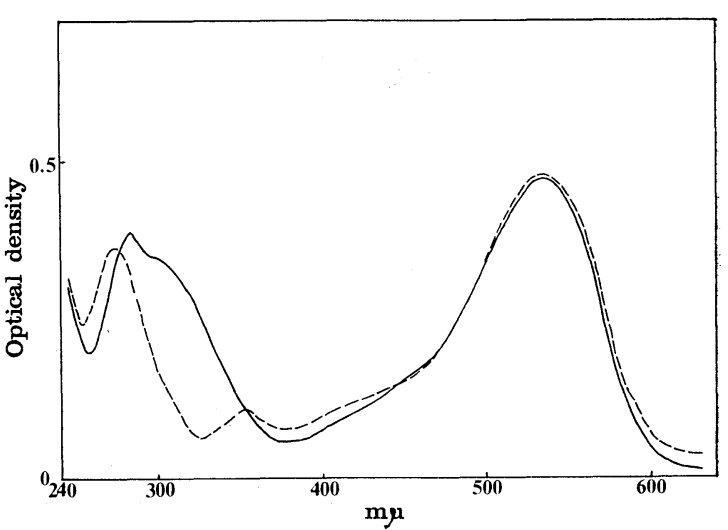

Fig. 1. Absorption Spectra. cayratinin, - - deacylated cayratinin. (in $\mathrm{MeOH}$ containing $\mathrm{HCl}$ in $0.05 \%$ ) hydroxyl of aglycone (delphinidin) is shown not only by oxidative degradation (detection of $p$-coumaroylsophorose) but also by comparison of absorption spectra of both delphin and awobanin. Similar difference in absorption maxima can also be recognized between delphin and awobanin. The absorption maxima (in $\mathrm{m} \mu$ ) are found at 261, 316, and 537 for awobanin chloride from Commelina $a^{2)}$ ), and at 260, 353, and 536 for delphin chloride (from blue Hyacint $h^{4)}$ ). Although the exact amounts of delphinidin triglucoside and $p$-coumaric acid in cayratinin have still to be determined, it is very likely that both components are present in the 1:1 ratio, when the absorption rates at the peaks (297 and $535 \mathrm{~m} \mu$ ) were carefully compared in Fig. 1 (refer to Ishikura and Hayashi ${ }^{77}$ ).

An exceeding similarity of absorption maxima of cayratinin and awobanin seems to indicate the linkage of the sugar residues with the 3-and 5-hydroxyl groups of delphinidin, since the ordinary 3-and 3,5-glucosides have the absorption maxima which are different from those of unusually substituted anthocyanins (refer to Harborne's information $\left.{ }^{9}\right)$.

The authors wish to express their sincere thanks to Prof. K. Hayashi of Tokyo Kyoiku University for providing the authentic sample of awobanin.

\section{References}

1) Kuroda, C., Bull. Chem. Soc. Japan 11: 265 (1936).

2) Mitsui, S., Hayashi, K., and Hattori, S., Bot. Mag. Tokyo 72 : 325 (1959).

3) Smith, I., Chromatographic Techniques, p. 194 (Smith, I., Ed., William HeinemannMedical Books. Ltd. London, 1958).

4) Shibata, M., and Ishikura, N., Seibutsu Kyoiku Kenkyu kiyo, 4 (1962).

5) Ishikura, N., and Hayashi, K., Bot. Mag.
Tokyo $78: 91$ (1965).

6) Bailey, R.W., J. Chromatography $8: 57$ (1962).

7) Shibata, M., Yamazaki, K., and Ishikura, N., Bot. Mag. Tokyo 78 : 299 (1965).

8) Ishikura, N., and Hayashi, K., Bot. Mag. Tokyo $76: 6$ (1963).

9) Harborne, J. B.. Comparative Biochemistry of the Flavonoids, p. 19 (Academic Press, London, 1965). 